\title{
Renal tubular dysgenesis with calvarial hypoplasia: report of two additional cases and review
}

\author{
Deborah E McFadden, J Tapio Pantzar, Margot I Van Allen, Sylvie Langlois
}

\begin{abstract}
We report two cases of renal tubular dysgenesis (RTD) with calvarial hypoplasia and review the originally reported cases of RTD that came from our institution and published reports regarding the association of RTD and skull abnormalities. Although previously reported in association with RTD, calvarial hypoplasia is probably under-recognised. The cases reported here support the idea that the skull abnormalities observed in the inherited form of renal tubular dysgenesis are a common component of the disorder, as they are in the acquired form of RTD associated with maternal use of ACE inhibitors. Renewed attention to this clinical manifestation of RTD may be important in suggesting the diagnosis before death, providing more complete information to parents and physicians facing important management decisions and ensuring appropriate pathological examination postmortem.

$(\mathcal{M}$ Med Genet 1997;34:846-848)
\end{abstract}

Keywords: renal tubular dysgenesis; calvarial hypoplasia; enlarged fontanelles

Renal tubular dysgenesis (RTD), first described in a pair of sibs in 1983 , is an autosomal recessive condition characterised by hypoplasia of the proximal tubules of the kidney, which may present with late onset oligohydramnios. ${ }^{12}$ Similar renal pathology is seen in some non-genetic conditions, characterised by renal hypoperfusion $^{3-5}$ and in infants born to women who took angiotensin converting enzyme inhibitors during pregnancy. ${ }^{6}$

In a review of reported cases of RTD, ${ }^{7}$ underdeveloped cranial bones or wide sutures and fontanelles were noted in $6 / 24$ cases. This finding was also present in other more recently reported cases..$^{8-10}$ We have examined two cases in which the presence of abnormally large fontanelles was particularly striking and was noted before death. This led us to review the originally reported cases. ${ }^{1}$

\section{Case reports}

CASE 1

A female infant was delivered at 32 weeks' gestational age to a gravida 2,26 year old woman, who had had one therapeutic abortion; she was not on any medication during pregnancy. The first and second trimesters of pregnancy were uncomplicated. An ultrasound examination performed at 13-14 weeks' gestational age (GA) was normal. At 32 weeks' GA, the woman presented with vaginal bleeding and mild abdominal pain. Ultrasound examination showed severe oligohydramnios with large, echogenic fetal kidneys. Spontaneous breech delivery occurred shortly thereafter. The infant had severe respiratory failure and profound hypotonia. The fontanelles were noted to be abnormally large. The diagnosis of Zellweger syndrome was considered.

The child died five hours after birth and necropsy was performed. Examination showed an infant with growth parameters at the 75th to 90th centile for 32 weeks' gestation and head circumference at the 25 th to 50 th centile. Although measurements were not recorded, the fontanelles were noted to be abnormally large with the anterior fontanelle extending into the middle of the forehead and the posterior fontanelle extending to just above the occipital bone; the sagittal suture was extremely wide. Grossly, the kidneys were large with a combined weight of $28.6 \mathrm{~g}$ versus an expected combined weight of $14.5 \pm 4.8 \mathrm{~g}$ and were somewhat spongy in appearance. Histologically, the kidneys showed diffuse glomerulocystic change and renal tubular dysgenesis characterised by absence of the proximal tubules as seen on routine microscopy and staining with PAS (present in the brush borders of proximal tubules) and epithelial membrane antigen (present within distal tubules). There was pulmonary hypoplasia as determined by volume measurement. Sedimentable catalase assay was normal and electron microscopic examination showed normal peroxisomes, excluding a diagnosis of Zellweger syndrome. Cytogenetic analysis showed a normal female karyotype. Neuropathological examination showed only rare small foci of heterotopic neurones; there were no morphological abnormalities to account for the hypotonia which was thus attributed to the respiratory failure.

\section{CASE 2}

This infant was delivered at 32 weeks' gestation to a gravida 2, para 1 woman who was said to have congenital deafness. Gestational diabetes had been diagnosed one week before delivery. There was no history of maternal drug use during pregnancy.

At birth, the infant had respiratory failure and was hypotonic. The skull was abnormal with large fontanelles. There were contractures at the knees and elbows with hyperflexibility at the ankles and wrists. A diagnosis of Zellweger syndrome was considered. The child died a few hours after birth.
Received 29 January 1997 Revised version accepted for publication 8 April 1997 
Necropsy showed a male infant with length at the 75 th to 90 th centile, weight at the 90 th centile, and head circumference at the 25 th to 50 th centile for a gestational age of 32 weeks. The fontanelles were very large, with the anterior confluent with the posterior, measuring 15 $\mathrm{cm} \times 6 \mathrm{~cm}$. There were prominent infraorbital creases, the thumbs were proximally implanted, and there were contractures, without pterygia, at the knees and elbows. The kidneys were large with a combined weight of $33.8 \mathrm{~g}$ versus an expected combined weight of $14.5 \pm$ $4.8 \mathrm{~g}$. The renal parenchyma was grossly normal. Histologically, there was renal tubular dysgenesis with absence of the proximal tubules. There was pulmonary immaturity with lung development appropriate for a gestational age of 28 weeks in contrast to the actual gestational age of 32 weeks. Analysis of very long chain fatty acids was normal as was the sedimentable catalase assay, excluding Zellweger disease. Cytogenetic analysis showed a normal male karyotype. Neuropathological examination showed extensive hypoxicischaemic damage which was thought to account for the hypotonia.

With the finding of markedly enlarged fontanelles in two infants found at necropsy to have renal tubular dysgenesis, we reviewed the necropsy reports of cases of RTD examined in our institution, including the two cases that were the subject of the initial report by Allanson et al. ${ }^{1}$ In case 1 from that report ( 34 week GA stillborn), the necropsy report states that "the sutures are very wide, the superior sagittal suture measuring $3 \mathrm{~cm}$ in width, with the large anterior and posterior fontanelle merging into each other". In the second case (36 week GA, stillborn), the report states "the skull bones are widely open, up to $2 \mathrm{~cm}$, the anterior fontanelle measures $5 \mathrm{~cm}$ in all dimensions". In a third case examined at our institution, the necropsy report noted that the anterior and posterior fontanelles were large, each measuring $3 \mathrm{~cm}$ in coronal and sagittal dimensions.

\section{Discussion}

The possibility of renal tubular dysgenesis may be raised when there is late onset of oligohydramnios, ${ }^{2}$ but the diagnosis is made only after histopathological examination. Large fontanelles or hypoplastic calvaria have been reported in a number of cases with renal tubular dysgenesis. In a review of reported cases of RTD, Allanson et al noted that six of 24 reported cases had associated microcephaly, underdeveloped cranial bones, or widely patent fontanelles. By history, one of the patients reported by Swinford et al had "extraordinarily wide cranial sutures and fontanelles" and the two cases initially reported by Allanson et $a l^{1}$ also had extremely large fontanelles, raising the proportion to nine of 24 . Since then, additional cases have been reported of calvarial hypoplasia in association with $\mathrm{RTD}^{8}{ }^{10}$; other reports note a normal skull ${ }^{11}$ while others fail to mention the skull at all. ${ }^{12}$ Our review of the original two cases indicates that abnormally large fontanelles or undermineralisation of the skull bones in RTD, as in ACE inhibitor fetopathy, are more common than reported and are often unrecognised or overlooked. ${ }^{6}$ These skull abnormalities appear to occur commonly enough in RTD that their presence could serve to raise the diagnosis clinically, followed by pathological confirmation. There are a number of conditions associated with enlarged fontanelles; for many, exclusion from the differential diagnosis is readily accomplished on the basis of the other clinical features. In both of our cases, a diagnosis of Zellweger syndrome was considered; the differential diagnosis for infants presenting in this fashion should also include RTD. Hypotonia in infants with RTD may occur on the basis of hypoxia owing to respiratory failure (pulmonary hypoplasia) and thus may not be seen in all cases of RTD as normal lung development has been reported. ${ }^{8}$

The basis for the skull abnormality in RTD is unknown. Similar renal and skull changes are seen in antenatal exposure to angiotensin converting enzyme inhibitors (ACEI fetopathy). It has been postulated that the renal and calvarial abnormalities reflect the effects of fetal hypotension induced by maternal ACEI ingestion with the resultant hypoxia affecting those organs requiring a high oxygen tension for normal growth. ${ }^{56}$ An alternative explanation is based on the finding that angiotensin II is a renal growth factor ${ }^{13}$ with the renal tubular abnormalities in ACE inhibitor fetopathy being attributed to lack of angiotensin II growth stimulation. The finding of calvarial hypoplasia in these cases has been interpreted as suggesting that calvarial bone growth may also be stimulated by angiotensin II. $^{14}$ In RTD, high levels of renal renin have been found and it has been suggested that an impaired feedback mechanism accounts for this. ${ }^{14}$ The possibility of this impaired feedback mechanism involving angiotensin receptors has been raised (though not formally examined) as the link between the high renin levels in the kidneys and the tubular dysgenesis; if there are abnormalities in angiotensin receptors in RTD kidneys there may be similar receptor abnormalities in the calvarium, providing the link between the renal and calvarial developmental abnormalities.

In conclusion, abnormalities of the skull occur fairly frequently in RTD and are often not recognised clinically, as is the case in ACE inhibitor fetopathy. The finding of enlarged fontanelles in an infant with oliguria, with or without respiratory failure or hypotonia, should serve to raise the possibility of RTD.

1 Allanson JE, Pantzar JT, MacLeod PM. Possible new autosomal recessive syndrome with unusual renal histopathological changes. Am F Med Genet 1983;16:57-60.

2 Swinford AAE, Bernstein J, Toriello HV, Higgins JV. Renal tubular dysgenesis: delayed onset of oligohydramnios. $A m \mathcal{F}$ Med Genet 1989;32:127-32.

3 Genest DR, Lage JM. Absence of normal-appearing proximal tubules in the fetal and neonatal kidney: prevalence and significance. Hum Pathol 1991;22:147-53.

4 Martin RA, Jones KL, Mendoza A, Barr M, Benirschke K. Effect of ACE inhibition on the fetal kidney: decreased renal blood flow. Teratology 1992;46:317-21.

5 Landing BH, Ang SM, Herta N, Larson, EF, Turner M. Labeled lectin studies of renal tubular dygenesis and renal atrophy of postnatal renal ischemia and end-stage kidney disease. Pediatr Pathol 1994;14:87-99. 
6 Barr M. Teratogen update: angiotensin converting enzyme inhibitors. Teratology 1994;50:399-409.

7 Allanson JE, Hunter AGW, Mettler GS, Jimenez C. Renal tubular dysgenesis: a not uncommon autosomal recessive syndrome. Am ₹ Med Genet 1992;43:811-14

8 Scarcella A, Pecoraro C, D'Agnello MR, Sole AN. Renal Scarcella A, Pecoraro C, D Agnello MR, Sole AN. Renal Nephrol 1994;8:216-17.

Metzman RA, Husson MA, Dellers EA. Renal tubular dysgenesis: a description of early maldevelopment in

0 Ariel I, Wells TR, Sagi M, Bar-Oz B, Ron N, Rosenmann E. Familial renal tubular dysgenesis: a disorder not isolated to proximal convoluted tubules. Pediatr Pathol Lab Med 1995; 15:915-22.

11 Bale PM, Kan AE, Dorney SFA. Renal proximal tubular dysgenesis associated with severe neonatal hemosiderotic liver disease. Pediatr Pathol 1994;14:479-89.

12 Moldavsky $M$, Shahin A, Turani H. Renal tubular dysgenesis present in a newborn with meconium ileus. dysgenesis present in a newborn with meconium ileus.

13 Wolf Wolf G, Neilson EG. Angiotensin II as a renal growth factor.
f Am Soc Nephrol 1993;3:1531-40.

4 Bernstein J, Barajas L. Renal tubular dysgenesis: evidence of abnormality in the renin-angiotensin sytem. $f \mathrm{Am}$ Soc Nephrol 1994;5:224-7. 